\title{
A New Flower Classification System Using LBP and SURF Features
}

\author{
Prashengit Dhar \\ Cox's Bazar City College, Cox's Bazar, Bangladesh \\ Email: nixon.dhar@gmail.com
}

Received: 06 October 2018; Accepted: 16 February 2019; Published: 08 May 2019

\begin{abstract}
Flowers are blessing of nature. Classification of flowers as a natural image is difficult as they are surrounded by background. So a segmentation phase is needed to separate the flower from background as good as possible. Computer vision has gained much attention for classification task. This paper proposes a method to classify flower with the help of LBP and SURF as features and SVM as a classifier. Input image is preprocessed for enhancement of image quality. Then the image is segmented by applying active contour segmentation method. After segmentation of the image, LBP and SURF features are extracted. SURF features are extracted from MSER regions. Then both features are concatenated. These concatenated features are sent for classification to SVM classifier. Quadratic SVM is employed here. Quadratic SVM trains these feature and tests to classify. We also tried out with different classifier. But they provide poor results. Proposed quadratic SVM achieves an accuracy of $87.2 \%$ which is significant and comparable for this classification taskK.
\end{abstract}

Index Terms-LBP, SURF, SVM, ROC, MSER, confusion matrix, active contour, segmentation

\section{INTRODUCTION}

Human likes flower. Different varieties of flowers are exist in this world. Remembering all the flowers is quite difficult. A flower classification system is useful in categorizing flower which may be known or unknown to a person. An unknown flower can be recognized by this proposed system. While travelling in different places we might be fall in front of different flowers that are unknown to us. At that moment a flower classification system can assist as a guide to be familiar with the flowers. This system can also be used in educational learning. Besides recognition if we could put some information about the flower so it can be a helping assistant. Learning with vision is much prettier and easy.

A natural flower image may contain different objects like leaves, sky, grasses or branches. So a pre-processing and segmentation is essential to eliminate these annoying objects as far possible. Before classification phase, here a segmentation technique is employed to remove background of the image. This technique uses an initial mask which is applied on the input image and evolves iteratively. Then the image is segmented in background and foreground. This is known active contour segmentation technique. Through this the flower is highlighted and background is removed as far as possible. After this, features are extracted and sent for classification. Computer vision has covered large area of classification. SVM provides good result in classification of image.

\section{RELATED WORK}

A variety of works have been done for flower classification. Moreover it is a popular topic now-a-days. Tiay proposed a flower recognition system that uses color features-red, blue, green hue, and saturation of color image [1]. Edge features are acquired by Hu's sevenmoment algorithm. KNN algorithm is used to classify. But use of color features may not work well in various environment. A segmentation algorithm developed by Angelova is based on identifying regions of potential object at detection stge. Then a Laplacian-based method of segmentation is applied guided by the initially detected regions [2]. Avishikta and Ranjan proposed a flower recognition system where segmentation is done by applying mask [3]. Mask is created by thresholding the image. Threshold value is computed from value channel of HSV. For recognition they used gist and color feature and svm classifier.

Wei and Liu and others suggest fusion descriptor based flower classification system [4].It's a new approach using nowadays. They used PHOW-HSV, Edge SIFT and PHOW-gray to extract color shape and texture respectively. These are used for fusion and SVM to classify. Krishna Singh proposed a method to represent the shape of the leaf by extracting twelve morphological features [5]. By applying these features they compared three plant classification techniques named probabilistic neural networks (PNN), Binary SVM Decision Tree (SVM-BDT) and Fourier moment technique. Amira and Asma extracted color features which are based on interest points of SURF [6]. Then Recognition is made by SVM. Their system performance was quite well. Gogul and Sathiesh developed a CNN based approach to recognize flower. Transfer learning method is used by them to extract features from flower species image [7].But it is time consuming. A segmentation approach proposed by 
Siraj and others on the basis of color clustering and flower's domain knowledge [8]. An approach proposed by Kulkarni which recognizes and identifies plants based on shape, texture and color features in combination with Zernike moments and Radial Basis Probabilistic Neural Networks (RBPNN) [9]. Nilsback and Zisserman proposed a classification approach on flower using vocabulary of shape, color and texture features [10].In this study, we have proposed a new approach to classify flower by the combination of LBP and SURF features. We have also shown the comparison of using features separately. Support Vector Machine is proposed here as a classifier.

\section{SYSTEM OVERVIEW}

This paper represents a flower classification system followed by segmentation and feature extraction. Input mage is processed first to remove noise and improve image quality. Noisy image can affects a system. Sometimes noisy image may lead to wrong segmentation. After that image is segmented by active contour segmentation method. This active contour segmentation method is iterative process. Though it is an iterative process, but the time taken by it is negligible. Then comes feature extract step. LBP and SURF features are separately extracted. Then a combination of both features is made. After that classification is performed. Quadratic SVM is applied to classify. The complete proposed system is depicted in fig. 1 .

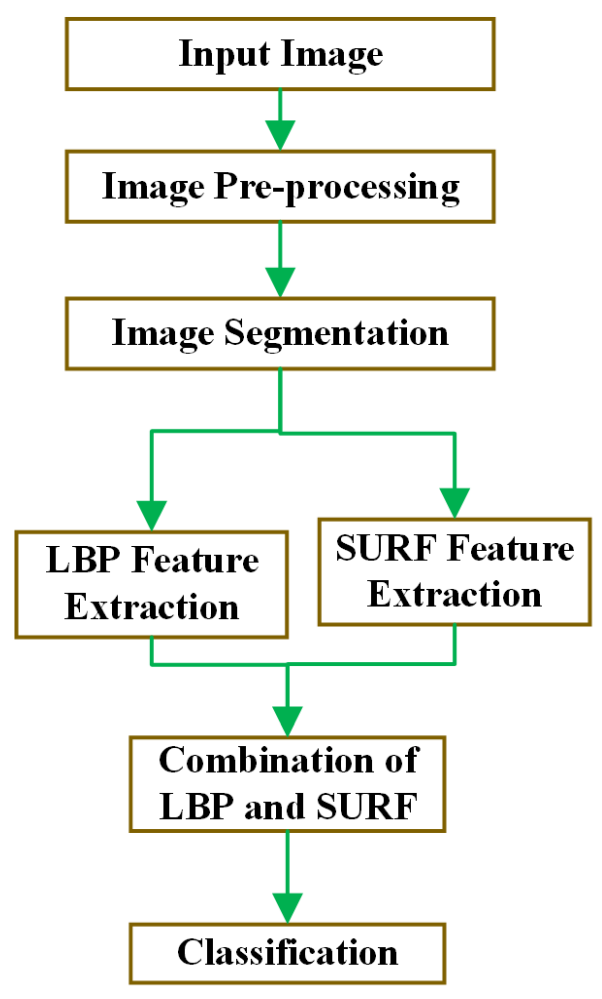

Fig.1. Proposed system

\section{DATASET}

Dataset is very crucial for classification task. It is very necessary to create a dataset on all possible environments. Benchmark dataset is very significant. In this study, Oxford17 flower dataset is considered. This dataset is benchmark. The dataset contains 17 types of flower and 80 samples for each class. 8 types of flower from the dataset are chosen. Selected flowers are shown in fig. 2. Flower image of the dataset are popular in UK. The selected flowers are - Daffodil, Iris, Fritillary, Sun flower, Daisy, Colts' foot, Windflower and Pansy.

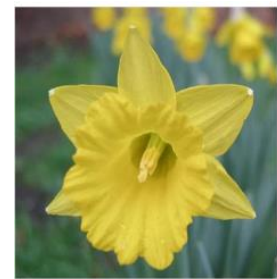

Daffodil

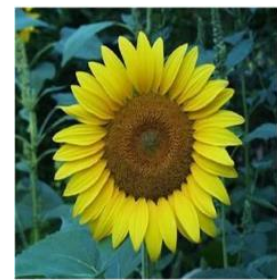

Sun Flower

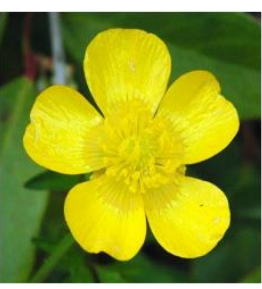

Buttercup

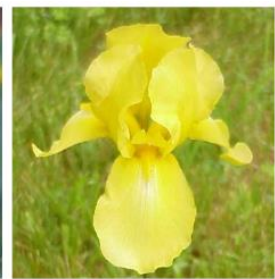

Iris

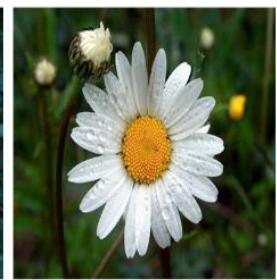

Daisy

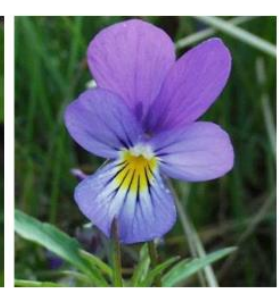

Pansy

Fig.2. Selected flower

\section{Segmentation}

Segmentation is a core phase in flower classification system. Flower image is segmented after pre-processing. Pre-processing makes the image finer for segmentation. Active contour segmentation is applied as segmentation technique. It is a process of iterative. This segmentation method segments the flower image into background and foreground. Sometimes it might be not possible to remove the background completely. This segmentation technique can segment image into background and foreground as much as possible. Flower mage may contain different background like- sky, leaf or any other thing. So it is mandatory to clear the background and 
focus on only the flower. Background objects affect negatively in classification. An initial mask is created for active contour segmentation. This mask defines a primary state of the active contour. Then segmentation is accomplished by evolving the active contour. The mask is a starting contour to begin the evolution of segmentation. This described active contour technique is based on chenVese method which involves no edges. The benefit of the method is that the contour is completely free so that it can either expand or shrink based on the features of image. Fig.3 shows example of segmentation.
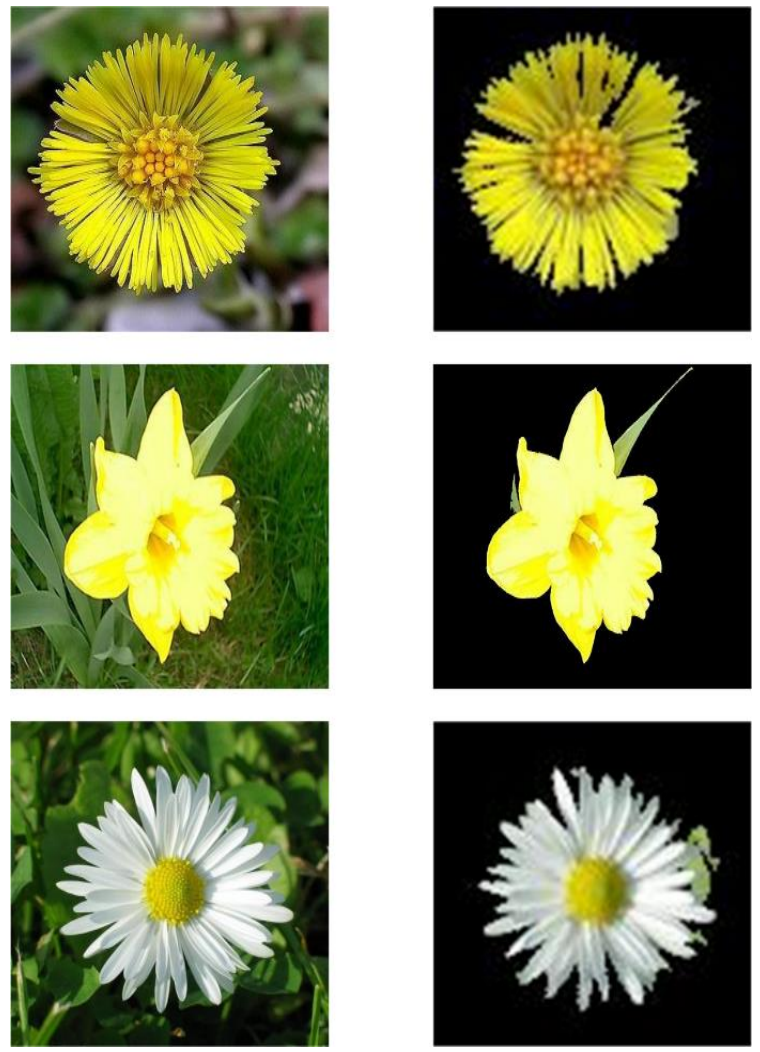

Fig.3. Before segmentation (left) and after segmentation (right)

\section{FEATURES}

\section{A. $L B P$}

Now in computer vision, a kind of graphical descriptor for classification is used which called Local Binary Patterns (LBP). LBP is that the observable case of Spectrum model of texture. Intended for classification of texture, it's since been initiate to a strong feature; it's more been determined that once LBP is joined with the Histogram of Oriented Gradients (HOG) descriptor, it improves the recognition performance expressively on some datasets. Fig.4 indications the three neighbourhood examples accustomed outline a texture and analyze a Local Binary Pattern (LBP).
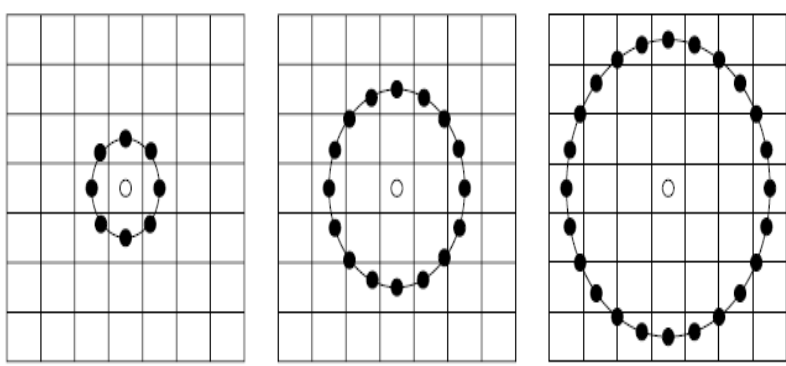

Fig.4. Neighborhood examples of LBP

\section{B. Maximally Stable Extremal Region}

MSER (Maximally Stable Extremal Region) could be a methodology for blob detection in pictures. The MSER algorithm extracts a variety of co-variant regions from picture, referred to as MSERs. Actually MSER itself is a steady connected element of some gray-level sets of the image. MSER relies on the concept of taking regions that keep nearly identical through a large variety of thresholds. All the pixels below a given threshold are stored as white and all those higher than or equal are stored as black. For a sequence of thresholded images, initially a black image will be appeared, then white spots which is corresponding to native intensity minima can seem then grow larger. These white spots can eventually merge, till the total image is white. The set of entire connected elements within the sequence is the set of entire extremal regions. Optionally, elliptical frames are connected to the MSERs by adjusting ellipses to the regions. Therefore those regions descriptors are features. The term extremal denotes to the fact that each one pixels within the MSER contains either lower or higher intensity than all the pixels on its bound. This time the system extracted strongest SURF features from MSER regions. Fig.5 visualizes MSER regions.

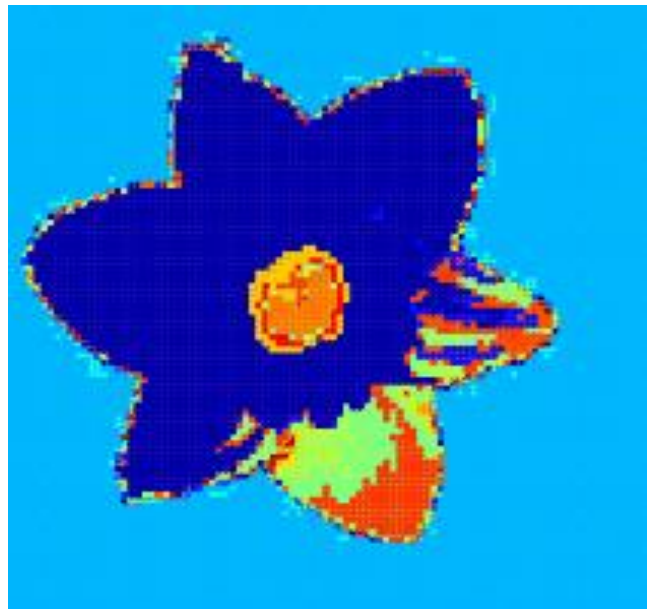

Fig.5. Visualizing MSER

\section{Speeded Up Robust Feature}


SURF (Speeded Up Robust Feature) is an extended version of SIFT algorithm. SURF is computationally faster than SIFT. Rotated object cannot make a negative effect while using SURF. This is a great advantage of SURF feature. Equal size of sub-regions is formed by dividing neighboring regions. For every region, a calculation of Haar-wavelet responses is made. Fig.6 shows extraction of surf feature. Different variations like occlusions, diverse illumination may exist in a flower image. In addition image may captured from numerous position. SURF can deliver compact and sound features to treat with these different variations. This system collected strongest features from the extracted feature vector.
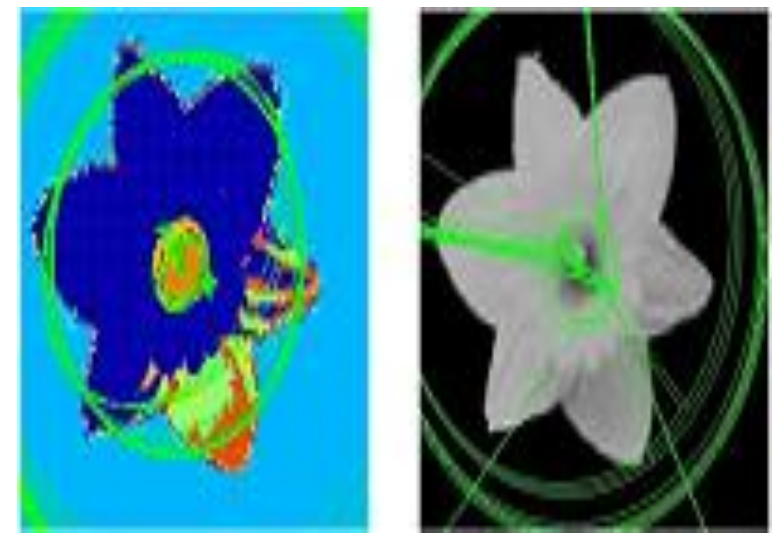

Fig.6. SURF feature extraction from MSER regions

\section{Combined Feature}

This phase is for combining both LBP and SURF features. LBP and SURF features are concatenated and creates only single feature vector. The resultant feature vector has 681 elements (531 LBP features and 150 strongest SURF features). This combined feature results in better performance for classification.

$$
F_{\text {Combine }}=\operatorname{concate}\left(F_{\text {LBP }}, F_{\text {SURF }}\right)
$$

\section{RECOGNITION}

Now at this recognition phase, flowers are classified into it's category. Recognition is supported by SVM classifier. Now SVM exists with a different variety like cubic, quadratic, Gaussian etc. Quadratic SVM is used in this system. SVM is well known for its classification based performance. A five fold cross validation took place, where one set is used for testing and remaining for training until 5 iterations. Matlab 2017a is used for simulation purpose.

\section{A. Performance Analysis}

Performance is analyzed by observing confusion matrix and ROC (Receiver Operating Characteristics) curve. Confusion matrix provides information about performance of the classifier.
In confusion matrix, each row refers to the instances in a genuine class and each column denotes the instances in a predicted class. Fig. 8 shows the confusion matrix of the proposed classifier. The true positive rate of the model is good enough to satisfy and false positive rate is tolerable. Besides ROC curve is stands with superior result. ROC illustrates the performance which is a graphical plot. The area under curve in ROC is closer or equal to 1 for all the classes which is notable and portrays effective output. Fig.7 to fig. 14 are ROC and fig.15 are confusion matrix of the classifier correspondingly.

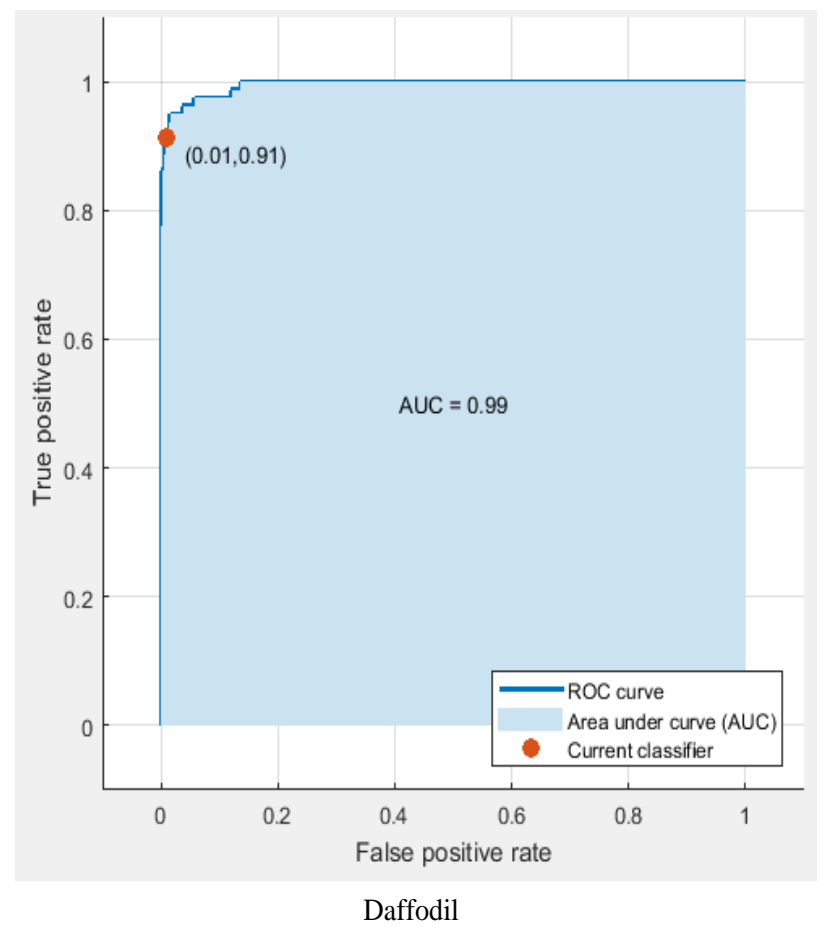

Fig.7. ROC of class- Daffodil

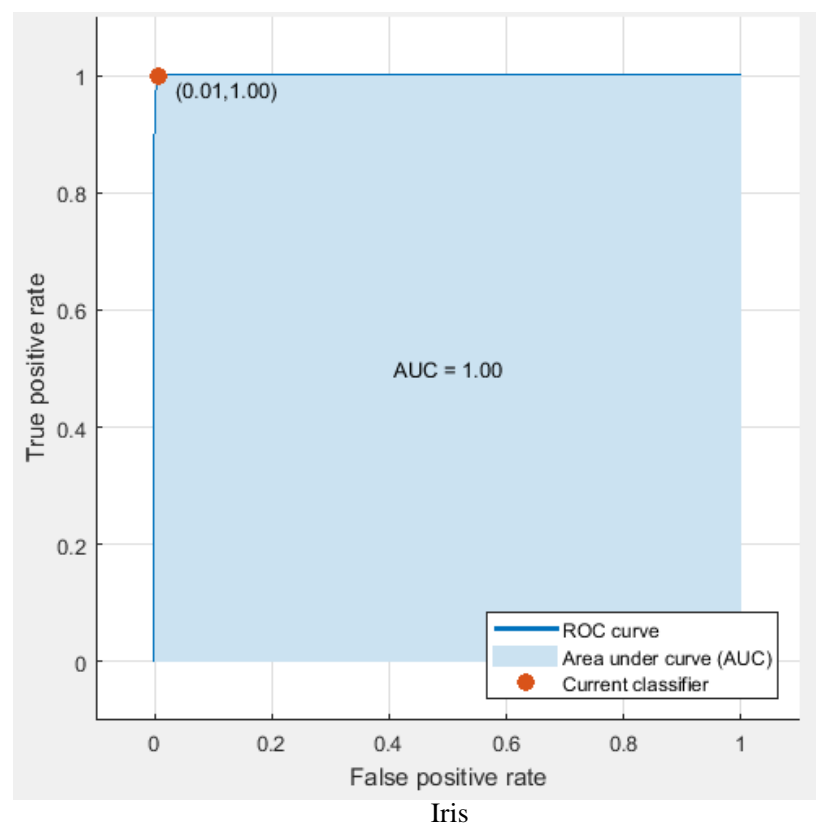

Fig.8. ROC of class- Iris 


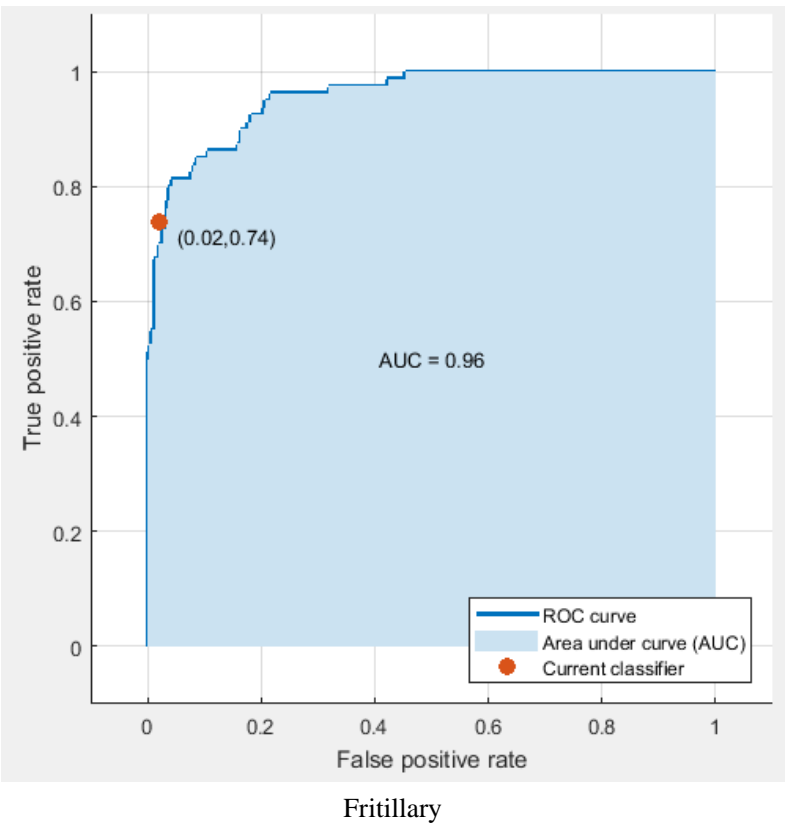

Fig.9. ROC of class- Fritillary

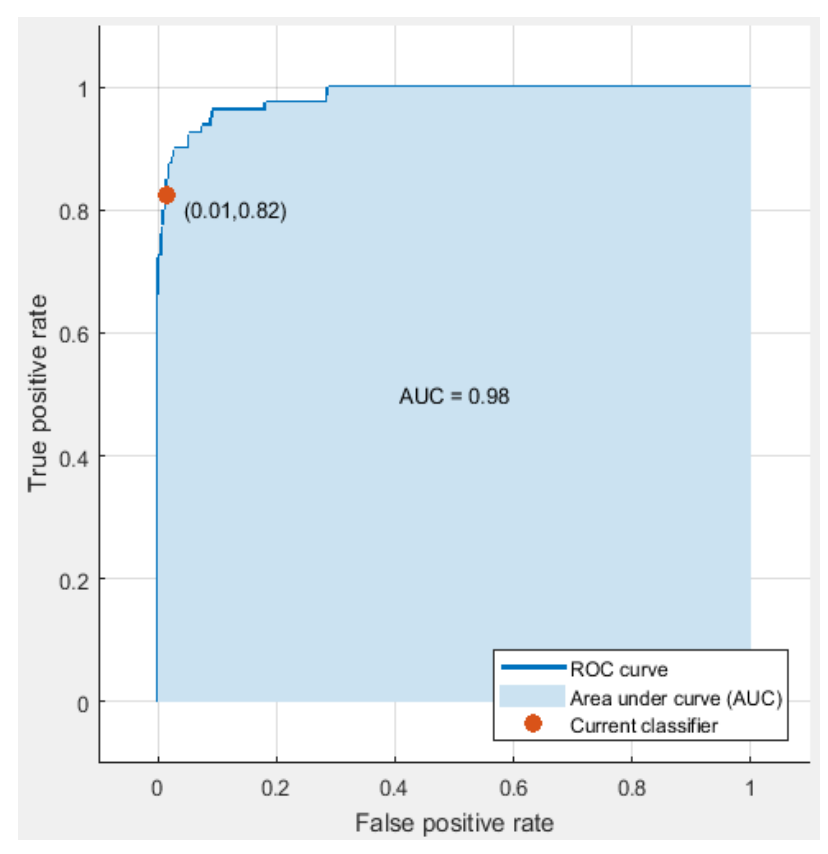

Sun Flower

Fig.10. ROC of class- Sun Flower

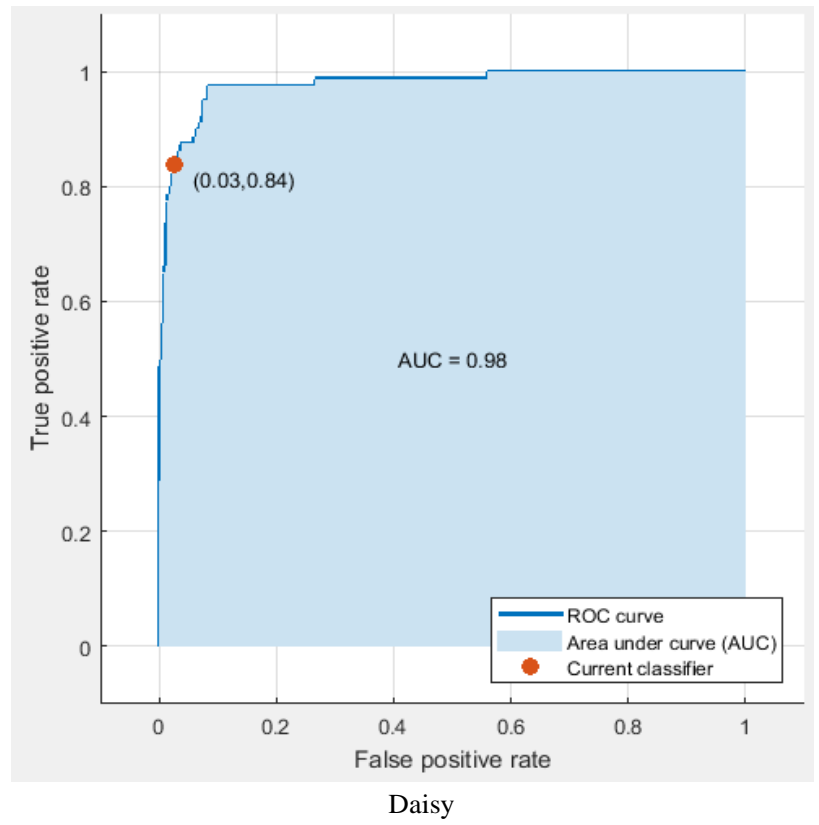

Fig.11. ROC of class- Daisy

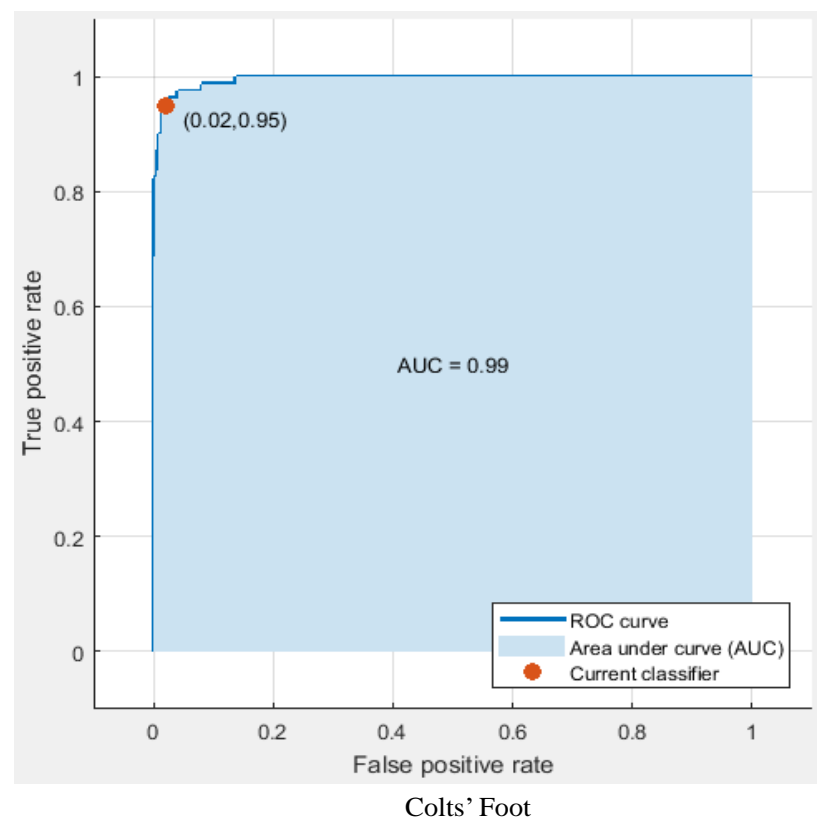

Fig.12. ROC of class - Colt's Foot 


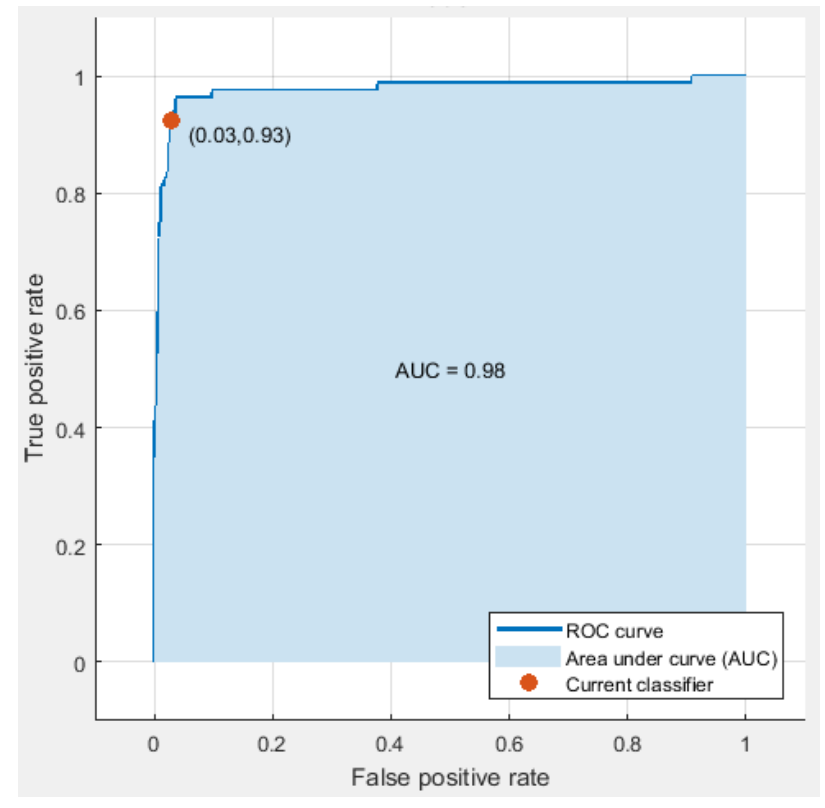

Wind Flower

Fig.13. ROC of class- Wind Flower

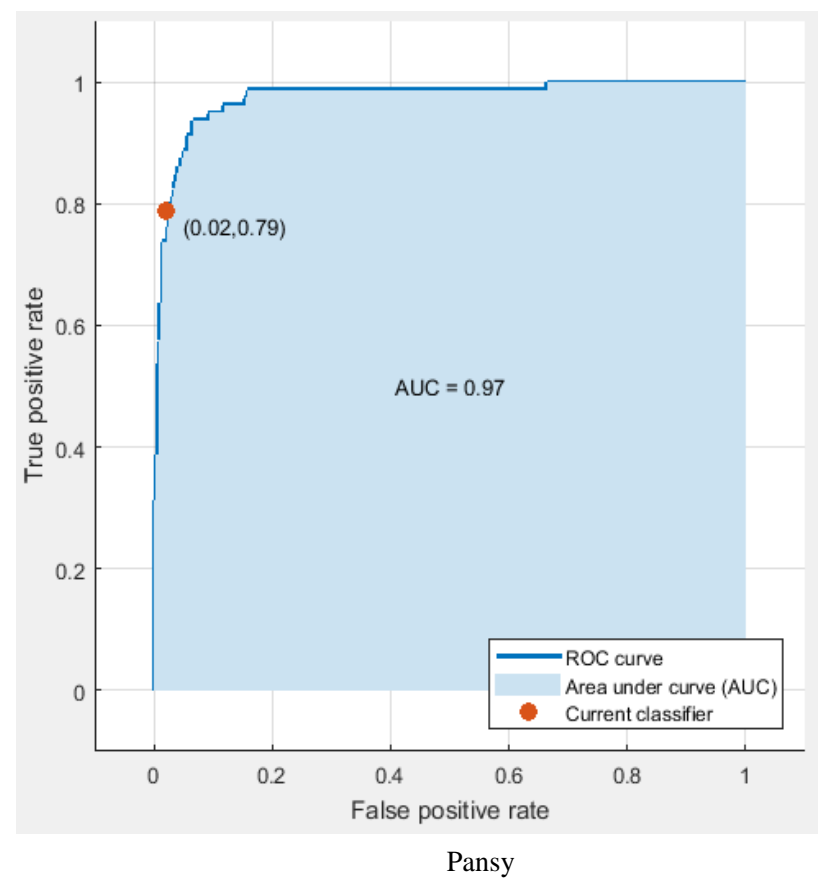

Fig.14. ROC of class-Pansy

\begin{tabular}{|c|c|c|c|c|c|c|c|c|}
\hline A & 73 & 1 & 5 & 1 & & & & \\
\hline B & & 80 & & & & & & \\
\hline C & 3 & 1 & 59 & 3 & 1 & 4 & 8 & 1 \\
\hline & 2 & & 2 & 66 & & 4 & 6 & \\
\hline & & & 1 & & 67 & & 2 & 10 \\
\hline $\mathrm{F}$ & 1 & & 2 & 1 & & 76 & & \\
\hline G & & & 2 & 2 & & 2 & 74 & \\
\hline $\mathrm{H}$ & & 1 & & 1 & 14 & 1 & & 63 \\
\hline & 4 & $\diamond$ & c & 0 & $\Leftrightarrow$ & 个 & $\sigma$ & 4 \\
\hline \multicolumn{9}{|c|}{ Predicted class } \\
\hline
\end{tabular}

(a)

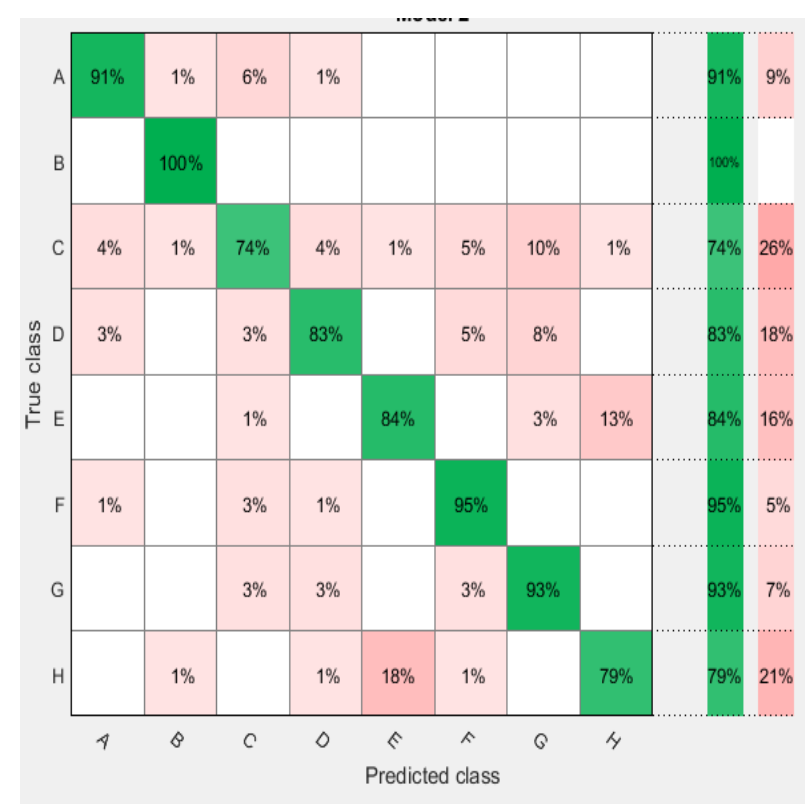

(b)

Fig.15. Confusion matrix ( $a$ and $b$ ) of the proposed classier

B. Comparison with other classifier 
This section is about to discuss and show contrast with different classifier. We have drawn a figure of contrast using features separately with other classifier. Table 1 depicts the contrast among different classification model using LBP feature. The feature dimension of LBP is 531*640. According to Table 1, SVM stretches good performance with respect to accuracy and decision tree is faster with respect to time. But no one is good enough to select. The term ObsPerSecond states to observation per second.

Table 1. LBP with different classifier

\begin{tabular}{|c|c|c|c|c|}
\hline Feature & Model & $\begin{array}{c}\text { Accuracy } \\
(\boldsymbol{\%})\end{array}$ & $\begin{array}{c}\text { Trainin } \\
\mathbf{g} \text { time(s) }\end{array}$ & $\begin{array}{c}\text { Prediction speed } \\
\text { (ObsPerSecond) }\end{array}$ \\
\hline LBP & $\begin{array}{c}\text { Quadratic } \\
\text { SVM }\end{array}$ & 82.2 & 9.6 & 760 \\
\hline LBP & KNN & 70.8 & 5.095 & 820 \\
\hline LBP & Adaboost & 69.4 & 134 & 630 \\
\hline LBP & $\begin{array}{c}\text { Decision } \\
\text { Tree }\end{array}$ & 55 & 12 & 1100 \\
\hline
\end{tabular}

While models are trained and tested for only SURF features, no good results are found. Here the SURF feature size is $150 * 640$, hence strongest features are selected. Classifiers provide miserable output. Table 2 demonstrates the output using SURF features for different classification model.

Table 2. SURF with different classifier

\begin{tabular}{|c|c|c|c|c|}
\hline Feature & Model & $\begin{array}{c}\text { Accuracy } \\
(\boldsymbol{\%})\end{array}$ & $\begin{array}{c}\text { Training } \\
\text { time(s) }\end{array}$ & $\begin{array}{c}\text { Prediction speed } \\
\text { (ObsPerSecond) }\end{array}$ \\
\hline SURF & $\begin{array}{c}\text { Quadratic } \\
\text { SVM } \\
\text { (Proposed) }\end{array}$ & 52.3 & 7.49 & 1100 \\
\hline SURF & KNN & 39.5 & 4.3651 & 1300 \\
\hline SURF & Adaboost & 45.2 & 47.433 & 920 \\
\hline SURF & $\begin{array}{c}\text { Complex } \\
\text { Tree }\end{array}$ & 35 & 6.37 & 2500 \\
\hline
\end{tabular}

The concatenation of both LBP features and SURF features delivers better accuracy in classification. The combined (LBP+SURF) feature length is $681 * 640$. Table 3 shows the statistics of merged features (LBP+SURR) performance with different classifiers. Among different classifiers, SVM outcomes with $87.2 \%$ accuracy which is proposed in this paper.

Table 3. LBP+SURF with different classifier

\begin{tabular}{|c|c|c|c|c|}
\hline Feature & Model & $\begin{array}{c}\text { Accuracy } \\
(\%)\end{array}$ & $\begin{array}{c}\text { Training } \\
\text { time(s) }\end{array}$ & $\begin{array}{c}\text { Prediction } \\
\text { speed } \\
\text { (ObsPerSeco } \\
\text { nd) }\end{array}$ \\
\hline LBP+SURF & $\begin{array}{c}\text { Quadratic } \\
\text { SVM } \\
\text { (Proposed) }\end{array}$ & $\mathbf{8 7 . 2}$ & $\mathbf{1 8 . 1 0 7}$ & $\mathbf{3 9 0}$ \\
\hline LBP+SURF & KNN & 72.8 & 8.442 & 410 \\
\hline LBP+SURF & Adaboost & 70.2 & 270 & 320 \\
\hline LBP+SURF & $\begin{array}{c}\text { Decision } \\
\text { Trees }\end{array}$ & 55 & 17.729 & 1300 \\
\hline
\end{tabular}

LBP provides outstanding texture descriptor of image and SURF features are robust to clutter, occlusion, scaling and rotation. As a response the combination achieves a significant result. In comparison with other system, the proposed model works better. A comparison with other model is given in Table 4.

Table 4. Comparison with other system

\begin{tabular}{|c|c|}
\hline System & Accuracy (\%) \\
\hline Proposed system & $87.2 \%$ \\
\hline$[3]$ & $85.93 \%$ \\
\hline
\end{tabular}

\section{CONCLUSION AND FUTURE WORKS}

This paper recommends a new flower classification system using combined LBP and SURF features and SVM is used as a classification model. Segmentation of flower proceeds ahead to get better classification result. Moreover this paper showed the contrast of using separate feature and different classifier. Using features distinctly results in inferior accuracy. Among all, using the combination of LBP and SURF features with SVM classifier demonstrates satisfactory result. In future we will try to attempt other dataset and to enlarge the quantity of classes. We will also try to explore some other features and classifier which can deliver improved result.

\section{ACKNOWLEDGMENT}

I wish to thank Dr. Rashed Mustafa for his support and help in this research.

\section{REFERENCES}

[1] T. Tiay, P. Benyaphaichit, P. Riyamongkol, "Flower Recognition System Based on Image Processing”, IEEE Third ICT International Student Project Conference, pp. 99-102, 2014

[2] A. Angelova, S. Zhu, Y. Lin, "Image segmentation for large-scale subcategory flower recognition", IEEE Workshop on Applications of Computer Vision (WACV), pp. 39-45. 2013.

[3] Avishikta Lodh, Ranjan Parekh, "Flower Recognition System based on Color and GIST Features",2017 Devices for Integrated Circuit (DevIC),pp.790-794

[4] Wei Liu, Yunbo Rao, Baijiang Fan, Jiali Song,Qifei Wang,"Flower Classification using Fusion Descriptor and SVM",Internation Smart Cities Conference 2017

[5] Krishna Singh, Indra Gupta, S. G., "Svm-bdt pnn and fourier moment technique for of leaf shape", International Journal of Signal Processing, Image Processing and Pattern Recognition (2010), pp.67-78.

[6] Amira Ben Mabrouk, Asma Najjar and Ezzeddine Zagrouba,"Image Flower Recognition based on a New Method for Color Feature Extraction", International Conference on Computer Vision Theory and Applications(2014),pp.201-206

[7] I.Gogul, V.Sathiesh Kumar,"Flower Species Recognition System using Convolution Neural Networks and Transfer Learning",International Conference on Signal Processing, Communications and Networking (ICSCN -2017) 
[8] Siraj, Fadzilah, Muhammad Ashraq Salahuddin, and Shahrul Azmi Mohd Yusof, "Digital Image Classification for Malaysian Blooming Flower", Computational Intelligence, Modelling and Simulation (CIMSiM), IEEE, 2010.

[9] A. H. Kulkarni, H. M. Rai, K. A. Jahagirdar and P. S. Upparamani, "A Leaf Recognition Technique for Plant Classification Using RBPNN and Zernike Moments", International Journal of Advanced Research in Computer and Communication Engineering (2013), Vol. 2, Issue 1, pp. 984-988.

[10] Nilsback and Andrew Zisserman, "A Visual Vocabulary for Flower Classification", Computer Vision and Pattern Recognition, IEEE Computer Society Conference on. Vol.2, 2006.

[11] X. Y. Wang, L. L. Liang and W. Y. Li, "A new SVMbased relevance feedback image retrieval using probabilistic feature and weighted kernel function", Journal of Visual Communication \& Image Representation, vol. 38, pp. 256-275, 2016.

[12] T. Hsu, C. Lee, L. Chen, "An interactive flower image recognition system", Springer, Multimedia Tools and Applications, Volume 53, Issue 1, pp 53-73, May 2011.

[13] Rashed Mustafa, Prashenjit Dhar," A Method to Recognize Food using Gist and SURF Features" 2018 Joint 7th International Conference on Informatics, Electronics \& Vision (ICIEV) and 2018 2nd International Conference on Imaging, Vision \& Pattern Recognition (icIVPR), pp:127-130.
[14] Hossam M. Zawbaa, Mona Abbass, Sameh H. Basha, Maryam Hazman, Abul Ella Hassenian,"An automatic flower classification approach using machine learning algorithms:, International Conference on Advances in Computing, Communications and Informatics (ICACCI), 2014.

[15] AnakornTiay, Pipimphorn Benyaphaichit, Panomkhawn Riyamongkol," Flower recognition system based on image processing", Third ICT International Student Project Conference (ICT-ISPC),2014, pp: $99-102$.

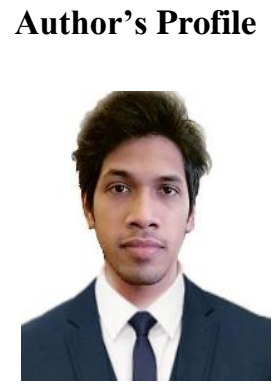

Prashengit Dhar received his B.Sc. degree in Computer Science and Engineering from University of Science and Technology Chittagong (USTC) and M.Sc. degree in Computer Science and Engineering from Port City International University. Currently he is working as a lecturer in a college. He has published many papers in conference and journal. His research interests include image processing, computer vision and machine learning

How to cite this paper: Prashengit Dhar, "A New Flower Classification System Using LBP and SURF Features", International Journal of Image, Graphics and Signal Processing(IJIGSP), Vol.11, No.5, pp. 13-20, 2019.DOI: 10.5815/ijigsp.2019.05.02 